\title{
AN ALGORITHM FOR FITTING MIXTURES OF GOMPERTZ DISTRIBUTIONS TO CENSORED SURVIVAL DATA
}

\section{G.J. MCLACHLAN ${ }^{1}$}

Centre for Statistics

Department of Mathematics

The University of Queensland

S.K. NG

Centre for Statistics

Department of Mathematics,

The University of Queensland

P. ADAMS

Department of Mathematics

The University of Queensland

D.C. MCGIFFIN

Department of Surgery

The University of Alabama at Birmingham

\section{A.J. GAILBRAITH,}

Department of Surgery

The Prince Charles Hospital

\footnotetext{
${ }^{1}$ Address for correspondence: Department of Mathematics, The University of Queensland, Queensland 4072, Australia.
} 


\section{PURPOSE AND DESCRIPTION}

We consider the fitting of a mixture of two Gompertz distributions to censored survival data. This model is therefore applicable where there are two distinct causes for failure that act in a mutually exclusive manner, and the baseline failure time for each cause follows a Gompertz distribution. For example, in a study of a disease such as breast cancer, suppose that failure corresponds to death, whose cause is attributed either to breast cancer or some other cause. In this example, the mixing proportion for the component of the mixture representing time to death from a cause other than breast cancer may be interpreted to be the cure rate for breast cancer (Gordon, 1990a and 1990b). This Gompertz mixture model whose components are adjusted multiplicatively to reflect the age of the patient at the origin of the survival time, is fitted by maximum likelihood via the EM algorithm (Dempster, Laird, and Rubin, 1977). There is the provision to handle the case where the mixing proportions are formulated in terms of a logistic model to depend on a vector of covariates associated with each survival time. The algorithm can also handle the case where there is only one cause of failure, but which may happen at infinity for some patients with a nonzero probability (Farewell, 1982).

\section{METHOD}

Let $T$ be a random variable denoting the failure time for a patient with an associated $p$-dimensional covariate vector $\boldsymbol{x}$ which contains at least the single covariate $w$. The density of $T$ is postulated to be the two-component mixture

$$
f(t ; \boldsymbol{x}, \boldsymbol{\Psi})=\sum_{i=1}^{2} \pi_{i}(\boldsymbol{x} ; \boldsymbol{\alpha}) f_{i}\left(t ; w, \boldsymbol{\theta}_{i}\right),
$$

where

$$
\begin{aligned}
f_{i}\left(t ; w, \boldsymbol{\theta}_{i}\right) & =h_{i}\left(t ; w, \boldsymbol{\theta}_{i}\right) \exp \left\{-\Lambda_{i}\left(t ; w, \boldsymbol{\theta}_{i}\right)\right\} \\
h_{i}\left(t ; w, \boldsymbol{\theta}_{\boldsymbol{i}}\right) & =\exp \left(\gamma_{i} w+\lambda_{i}+\beta_{\boldsymbol{i}} t\right) \\
\Lambda_{i}\left(t ; w, \boldsymbol{\theta}_{\boldsymbol{i}}\right) & =e^{\gamma_{i} w} e^{\lambda_{i}}\left(e^{\beta_{i} t}-1\right) / \beta_{\boldsymbol{i}},
\end{aligned}
$$

and

$$
\boldsymbol{\theta}_{i}=\left(\lambda_{i}, \beta_{i}, \gamma_{i}\right)^{\prime}
$$

for $i=1,2$, and where

$$
\Psi=\left(\boldsymbol{\alpha}^{\prime}, \boldsymbol{\theta}_{1}^{\prime}, \boldsymbol{\theta}_{2}^{\prime}\right)^{\prime}
$$

Here a prime denotes vector transpose.

The mixing proportion $\pi_{i}(\boldsymbol{x} ; \boldsymbol{\alpha})$ is modelled to depend on $\boldsymbol{x}$ through the logistic model for which

$$
\begin{aligned}
\pi_{1}(\boldsymbol{x} ; \boldsymbol{\alpha}) & =1-\pi_{2}(\boldsymbol{x} ; \boldsymbol{\alpha}) \\
& =e^{\boldsymbol{a}+\boldsymbol{b}^{\prime} \boldsymbol{x}} /\left(1+e^{\left.\boldsymbol{a}+\boldsymbol{b}^{\prime} \boldsymbol{x}\right)}\right.
\end{aligned}
$$


where $\boldsymbol{\alpha}=\left(a, \boldsymbol{b}^{\prime}\right)^{\prime}$ is the parameter vector.

It can be seen from (3) that the hazard function corresponding to the $i$ th component of the mixture has the form

$$
h_{i}\left(t ; w, \boldsymbol{\theta}_{i}\right)=e^{\gamma_{i} w} h_{i, 0}\left(t ; \boldsymbol{\theta}_{i}\right),
$$

where the baseline hazard function $h_{i, 0}\left(t ; \boldsymbol{\theta}_{i}\right)$ is represented by the Gompertz model, for which

$$
h_{i, 0}\left(t ; \boldsymbol{\theta}_{i}\right)=\exp \left(\lambda_{i}+\beta_{i} t\right) \quad(i=1,2) .
$$

Hence the covariate $w$ is modelled to have an additive effect on the $i$ th component hazard function on the $\log$ scale. Often in practice, $w$ will be the age of the patient at the instance from which the time to failure is being measured.

For the $j$ th entity $(j=1, \ldots, n)$, we observe

$$
\boldsymbol{y}_{j}=\left(t_{j}, \boldsymbol{x}_{j}^{\prime}, \delta_{1 j}, \delta_{2 j}, \delta_{3 j}\right)^{\prime},
$$

where $\delta_{1 j}, \delta_{2 j}$ and $\delta_{3 j}$ are zero-one indicator variables with $\delta_{i j}=1$ if entity $j$ failed due to cause $i$ and zero otherwise $(i=1,2)$, and $\delta_{3 j}=1$ if entity $j$ was censored at time $t_{j} ; \boldsymbol{x}_{j}$ is the covariate vector recorded on the $j$ th entity $(j=1, \ldots, n)$. Thus

$$
\delta_{1 j}+\delta_{2 j}+\delta_{3 j}=1
$$

for $j=1, \ldots, n$. As above, we let $\boldsymbol{\Psi}=\left(\boldsymbol{\alpha}^{\prime}, \boldsymbol{\theta}_{1}^{\prime}, \boldsymbol{\theta}_{2}^{\prime}\right)^{\prime}$ be the vector containing all the unknown parameters. Then the $\log$ likelihood for $\boldsymbol{\Psi}$ formed on the basis of $\boldsymbol{y}_{1}, \ldots, \boldsymbol{y}_{n}$ is given by

$$
\begin{aligned}
\log L(\boldsymbol{\Psi})= & \sum_{j=1}^{n}\left[\sum_{i=1}^{2} \delta_{i j} \log \left\{\pi_{i}\left(\boldsymbol{x}_{j} ; \boldsymbol{\alpha}\right) f_{i}\left(t_{j} ; w_{j}, \boldsymbol{\theta}_{\boldsymbol{i}}\right)\right\}\right. \\
& \left.+\delta_{3 j} \log \sum_{i=1}^{2}\left\{\pi_{\boldsymbol{i}}\left(\boldsymbol{x}_{j} ; \boldsymbol{\alpha}\right) S_{\boldsymbol{i}}\left(t_{j} ; w_{j}, \boldsymbol{\theta}_{i}\right)\right\}\right]
\end{aligned}
$$

where

$$
S_{i}\left(t ; w, \boldsymbol{\theta}_{i}\right)=\exp \left\{-\Lambda_{i}\left(t ; w, \boldsymbol{\theta}_{i}\right)\right\}
$$

is the $i$ th component survival function.

The parameter vector $\boldsymbol{\Psi}$ is estimated by maximum likelihood. The likelihood equation

$$
\partial \log L(\boldsymbol{\Psi}) / \partial \Psi=\mathbf{0}
$$

is solved via the EM algorithm of Dempster et al. (1977). For those entities with censored observations (that is, $\delta_{3 j}=1$ ), we introduce the zero-one indicator vector, $\boldsymbol{z}_{j}=\left(z_{1 j}, z_{2 j}\right)^{\prime}$, where $z_{i j}=1$ or 0 according as entity $j$ would have failed from cause $i$ or not $(i=1,2)$. The EM algorithm is applied with the $\boldsymbol{y}_{j}$ and the $\boldsymbol{z}_{j}$ viewed as the complete-data. The actual time to failure for those entities with $\delta_{3 j}=1$ was not 
introduced as an incomplete variable in the complete-data framework, as it did not simplify the calculations.

It follows on application of the EM algorithm in the aforementioned framework that on the $(k+1)$ th iteration, the estimate of $\boldsymbol{\Psi}, \Psi^{(k+1)}$, is the maximizer of the function

$$
\begin{aligned}
Q\left(\Psi ; \Psi^{(k)}\right)=\sum_{j=1}^{n} & \sum_{i=1}^{2}\left[\delta_{i j} \log \left\{\pi_{i}\left(\boldsymbol{x}_{j} ; \boldsymbol{\alpha}\right) f_{i}\left(t_{j} ; w_{j}, \boldsymbol{\theta}_{i}\right)\right\}\right. \\
& \left.+\delta_{3 j} \tau_{i}\left(t_{j} ; \boldsymbol{x}_{j}, \mathbf{\Psi}^{(k)}\right) \log \left\{\pi_{i}\left(\boldsymbol{x}_{j} ; \boldsymbol{\alpha}\right) S_{\boldsymbol{i}}\left(t_{j} ; w_{j}, \boldsymbol{\theta}_{i}\right)\right\}\right],
\end{aligned}
$$

where $\boldsymbol{\Psi}^{(k)}$ is the estimate of $\boldsymbol{\Psi}$ on the previous $k$ th iteration and

$$
\tau_{i}\left(t_{j} ; \boldsymbol{x}_{j}, \Psi\right)=\pi_{i}\left(\boldsymbol{x}_{j} ; \boldsymbol{\alpha}\right) S_{i}\left(t_{j} ; w_{j}, \boldsymbol{\theta}_{i}\right) / \sum_{h=1}^{2} \pi_{h}\left(\boldsymbol{x}_{j} ; \boldsymbol{\alpha}\right) S_{h}\left(t_{j} ; w_{j}, \boldsymbol{\theta}_{h}\right)
$$

is the posterior probability that the $j$ th entity with censored survival time $t_{j}$ would have failed due to cause $i(i=1,2)$.

Unfortunately, for Gompertz component distributions, $\Psi^{(k+1)}$ does not exist in closed form, as discussed in McLachlan and Krishnan (1997). In our algorithm, it is computed iteratively, commencing with $\boldsymbol{\alpha}^{(k+1)}$, since its calculation does not involve $\boldsymbol{\theta}_{1}^{(k+1)}$ and $\boldsymbol{\theta}_{2}^{(k+1)}$. The estimate $\boldsymbol{\alpha}^{(k+1)}$ is computed iteratively by NewtonRaphson. The computation of $\boldsymbol{\theta}_{1}^{(k+1)}$ and $\boldsymbol{\theta}_{2}^{(k+1)}$ is then undertaken iteratively using a quasi-Newton method. As noted by Dempster et al. (1977), it is not essential that $\Psi^{(k+1)}$ globally maximizes $Q\left(\Psi ; \Psi^{(k)}\right)$ for the monotonicity property of the sequence of iterates $\left\{\Psi^{(k)}\right\}$ to hold. A sufficient condition is

$$
Q\left(\boldsymbol{\Psi}^{(k+1)}, \boldsymbol{\Psi}^{(k)}\right) \geq Q\left(\boldsymbol{\Psi}^{(k)}, \boldsymbol{\Psi}^{(k)}\right)
$$

The use of (6) as a means of choosing $\Psi^{(k+1)}$ corresponds to using the generalized EM algorithm (Dempster et al. 1977).

In order to improve the speed of convergence, an E-step is performed in our program after the computation of $\boldsymbol{\alpha}^{(k+1)}$ and before the computation of the other subvectors $\boldsymbol{\theta}_{1}^{(k+1)}$ and $\boldsymbol{\theta}_{2}^{(k+1)}$ in $\boldsymbol{\Psi}^{(k+1)}$. In the terminology of Meng and Rubin (1993), it can be viewed as applying a multicycle ECM (expectation conditional-maximization) algorithm, where a cycle is defined to be an E-step followed by a CM-step. This multicycle E-step is effected here by updating $\boldsymbol{\alpha}^{(k)}$ by $\boldsymbol{\alpha}^{(k+1)}$ in $\boldsymbol{\Psi}^{(k)}$ in the righthand side of (5). A multicycle ECM algorithm is not necessarily a GEM algorithm. However, it does increase the likelihood function at each cycle, and hence at each iteration (Meng and Rubin, 1993). To further enhance convergence in our program, another E-step is performed after the computation of $\boldsymbol{\alpha}^{(k+1, m+1)}$ for each $m$. This may affect the monotone convergence of the consequent sequence of likelihood values. In our particular applications the monotone convergence was preserved.

The standard errors of the maximum likelihood estimator $\hat{\boldsymbol{\Psi}}$ of $\boldsymbol{\Psi}$ is assessed using the bootstrap methodology of Efron $(1979,1982)$. A number $K$ (as specified by 
the user) of independent bootstrap samples are obtained with each being randomly drawn with replacement from the observed data $\boldsymbol{y}_{1}, \ldots, \boldsymbol{y}_{n}$.

The program can also handle the case where there is only one cause of failure, but which may not happen for some entities; that is, there is a nonzero probability that the time to failure is infinity. In this case, $f_{1}\left(t_{j} ; w_{j}, \boldsymbol{\theta}_{1}\right)$ and $S_{1}\left(t_{j} ; w_{j}, \boldsymbol{\theta}_{1}\right)$ are each set identically equal to 1 . The mixing proportion $\pi_{1}\left(\boldsymbol{x}_{j} ; \boldsymbol{\alpha}\right)$ now represents the prior probability that an entity with covariate vector $\boldsymbol{x}_{j}$ will not fail (that is, will have an infinite failure time).

\section{ABOUT THE SOFTWARE}

The software is written in FORTRAN77. To compile the algorithms, several steps are required to be carried out which are listed in the file "makefile.txt". The main algorithm is named MGOMP in which the subroutine CHOL (algorithm AS6) and SYMINV (algorithm AS7) are called to invert a positive definite symmetric matrix. To generate random numbers for the bootstrap application, the subroutine RANDOM (algorithm AS183) is called to return a pseudo-random integer taken from a uniform distribution between 0 and 1 , excluding the end points. In addition, the MINPACK routine HYBRD1 (Moré et al.,1980) is called to find a solution of a system of two nonlinear equations. Thus, there are five FORTRAN files named MGOMP.f, CHOL.f, SYMINV.f, RANDOM.f, and HYBRID.f in the package.

To run the software, two input files are required. The first file "para.dat" provides the initial estimates for the parameters $\lambda_{i}, \beta_{i}, \gamma_{i}(i=1,2), a$ and the coefficient $b_{1}$ of $w$. The other elements in the coefficient $\boldsymbol{b}$ of $\boldsymbol{x}$ are set initially to zero. The second file "surv.dat" contains the data $\boldsymbol{y}_{j}=\left(t_{j}, w_{j}, \delta_{1 j}, \delta_{2 j}, \boldsymbol{x}_{j}^{\prime}\right)^{\prime}$ of each observation, where $t_{j}, w_{j}, \boldsymbol{x}_{j}$, and $\delta_{i j}(i=1,2)$ are defined as in the previous section.

For the sake of achieving satisfactory accuracy on the parameter estimates, doubleprecision is used in the algorithm. The stopping criterion for convergence is based on the absolute relative changes in the parameters, and a value of $10^{-4}$ is used for the cumulative (absolute) relative change. The final estimates of the parameters are given in the output file "fort.25".

The structure of the subroutine GETEST is given below. It defines the input and output variables in the algorithms. 
SUBROUTINE GETEST(NBOOT,MODEL,PI2EQ1,SIZE,FTIME,AGE,DELTA, NUMCOV,COV,ESTIN,ESTOUT,LLOUT,COEOUT,ITER,IFAIL,CODE)

$\begin{array}{ll}\text { NBOOT } & \text { Integer } \\ \text { MODEL } & \text { Integer } \\ & \\ \text { PI2EQ1 } & \text { Integer } \\ & \\ \text { SIZE } & \begin{array}{l}\text { Integer } \\ \text { FTIME }\end{array} \\ & \begin{array}{l}\text { Double precision } \\ \text { array (SIZE) } \\ \text { Double precision } \\ \text { array (SIZE) }\end{array} \\ \text { DELTA } & \begin{array}{l}\text { Integer } \\ \text { array (3,SIZE) }\end{array}\end{array}$

NUMCOV Integer

COV Double precision array

(MAXCOV,SIZE)

ESTIN Double precision array (8)

ESTOUT Double precision array $(\mathrm{MB}, 8)$

LLOUT Double precision array (MB)

COEOUT Double precision array (MB,MAXCOV)

ITER Integer

IFAIL Integer

CODE Integer input: bootstrap iterations indicator:

$=1$ indicate the complete data is used;

$\neq 1$ indicate the number of bootstrap iterations needed.

input: model indicator:

$=1$ if model with two distinct causes of failure;

$=2$ if only one cause of failure is considered.

input: mixing proportion indicator:

$=0$ if fitting two components of Gompertz distributions;

$=1$ if fitting single Gompertz distribution $\left(\pi_{2 j}=1 \forall j\right)$.

input: the number of entities in the data set.

input: the failure (or censoring) time of each of the entities.

input: the age of each of the entities at the time. of operation, that is $w_{j}$

input: zero-one indicator: $d(1, j)=1$ if entity $j$ failed due to Cause 1 ; $d(2, j)=1$ if entity $j$ failed due to Cause 2 ; $d(3, j)=1$ if entity $j$ was censored.

input: the number of additional covariates besides AGE to be included in the logistic model.

input: the entries $(l, j)(l=1, \ldots, N U M C O V)$ give the values for the $l$ th covariate of the $j$ th entity, MAXCOV $=20$ is the maximum number of covariates.

input: the initial estimates for the parameters:

$\lambda_{1}, \beta_{1}, \gamma_{1}, \lambda_{2}, \beta_{2}, \gamma_{2}, a$ and $b_{1}$.

output: the final estimates for the parameters for each bootstrap iteration $k(k=1, \ldots, N B O O T)$, $\mathrm{MB}=100$ is the maximum number of bootstraps.

output: the final estimates for each of calculated loglikelihoods for each bootstrap iteration.

output: the entries $(k, l)(k=1, \ldots, N B O O T)$ give the final estimate of the coefficient for the $l$ th extra covariate in the $k$ th bootstrap.

output: the number of iterations made in estimation.

output: fault indicator:

$=0$ if no error is detected;

$=1$ if input or model-specification error is detected;

$=2$ if error is detected in estimation of $a \& b_{1}$;

$=3$ if error is detected in estimation of $\boldsymbol{\theta}_{1} \& \boldsymbol{\theta}_{2}$.

output: failure code for fault indicator IFAIL $=2$ or 3 :

$=0$ if no error is detected;

$=1$ if error is detected in matrix inversion (for IFAIL=2);

$=2$ if suspected non-termination of $a \& b_{1}$ (for IFAIL=2);

$=$ IFAULT (for IFAIL $=3$ ); see the description in the subroutine HYBRD1 of file "HYBRID.F". 


\section{EXAMPLE}

As an illustration, the Stanford heart transplant data (Crowley and Hu, 1977) are analysed, using this algorithm to fit a Gompertz mixture model. These data have been analysed also by Larson and Dinse (1985) and Kuk (1992). We consider only the subset of 65 patients who received a transplant and who had complete data on the covariates of interest: mismatch score and age at transplant. Deaths were attributed to transplant rejection (Group 1) or other causes (Group 2). Among the 65 transplant recipients, there were 29 rejection deaths, 12 deaths from other causes, and 24 censored observations. Here the covariates are transformed to have zero means and unit variances. We include both covariates in the logistic model and the age at transplant in the component Gompertz distributions. The standard errors of the maximum likelihood estimates are obtained by bootstrap resampling with $K=100$ replications. The results are presented in Table 1.

Table 1: Maximum likelihood estimates (standard errors) for two-component Gompertz mixture model

\begin{tabular}{lrrr}
\hline & Logistic model & \multicolumn{2}{c}{ Component parameters } \\
& & Rejection death & Other causes \\
Covariate & MLE (S.E.) & MLE (S.E.) & MLE (S.E.) \\
\hline Constant & $1.396(0.499)$ & & \\
Mismatch score & $0.358(0.746)$ & & \\
Age at transplant & $0.303(0.556)$ & $1.023(0.346)$ & $0.275(0.688)$ \\
$\lambda$ & & $-6.335(0.428)$ & $-3.927(0.742)$ \\
$\beta$ & & $-0.0015(0.0007)$ & $-0.0055(0.0124)$ \\
\hline
\end{tabular}


CODE OF THE ALGORITHM MGOMP.F

PROGRAM MGOMP

C

*******************************************************************

** Purpose: To fit a mixture of two Gompertz distributions **

** to censored survival data

**Input files: (1) data file "surv.dat";

**

**Output file: final estimates file "fort.25"

integer MB, MAX, MAXCOV

parameter $(M B=100)$

parameter $(\mathrm{MAX}=5000)$

parameter (MAXCOV=20)

integer nboot, model, pi2eq1, size, delta( $3, \mathrm{MAX})$, numcov, ifail

integer iter, code

double precision ftime(MAX), age(MAX), cov(MAXCOV, MAX), estin(8)

double precision estout (MB, 8), llout (MB), coeout (MB, MAXCOV)

C

CALL init(nboot,model, pi2eq1, size,ftime, age, delta, numcov,

+ cov,estin, ifail)

if (ifail.ne.0) goto 200

CALL getest (nboot, model, pi2eq1, size, ftime, age, delta, numcov,

+ cov, estin, estout, llout, coeout, iter, ifail, code)

CALL output (nboot, size, numcov, estin, estout, llout, coeout,

+ iter, ifail, code)

stop

end

SUBROUTINE init (nboot, model, pi2eq1, size,ftime, age, delta, numcov,

$+\operatorname{cov}$, estin, ifail)

C

$\mathrm{C}$

C

C

** To get data, initial estimates and model specification **

$* * * * * * * * * * * * * * * * * * * * * * * * * * * * * * * * * * * * * * * * * * * * * * * * * * * * * * * * * * * * * * * * * * *$

integer MB, MAX, MAXCOV

parameter $(\mathrm{MB}=100)$

parameter $(\mathrm{MAX}=5000)$

parameter $(\mathrm{MAXCOV}=20)$

common/randc/ix, iy, iz

integer ix,iy,iz

integer nboot,model, pi2eq1, size,delta(3,MAX), totcov, ifail

double precision ftime(MAX), age(MAX), cov(MAXCOV, MAX), estin(8)

double precision tempc(MAXCOV,MAX)

character*1 userin

open (21,file='surv.dat')

open (22,file='para.dat')

if ail=1

print *,'Please select an option: [c] for continue'

print *,'

[q] for quit,

read *, userin

if (userin( $1: 1$ ).eq. 'q') goto 900

print *,', 


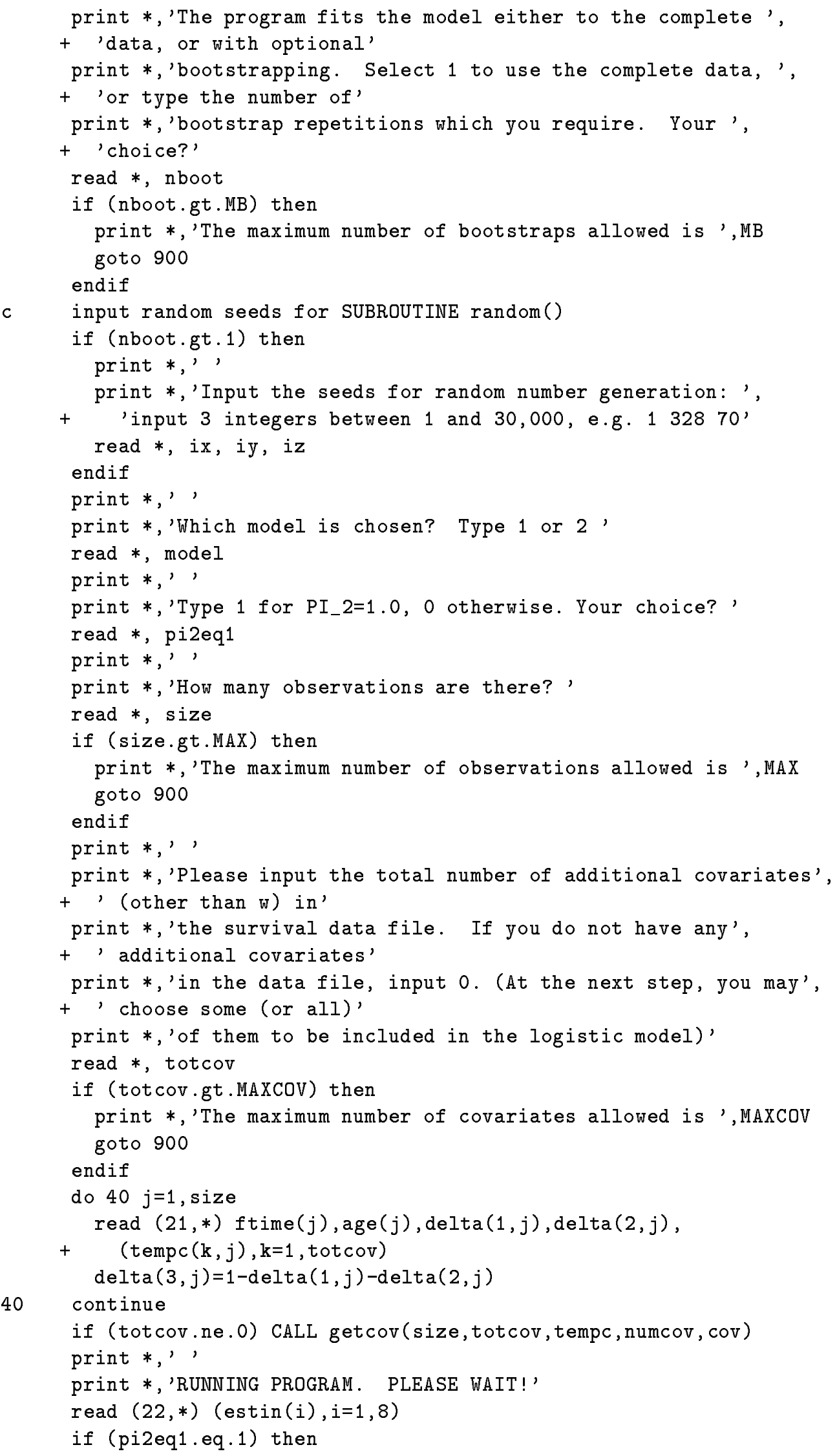




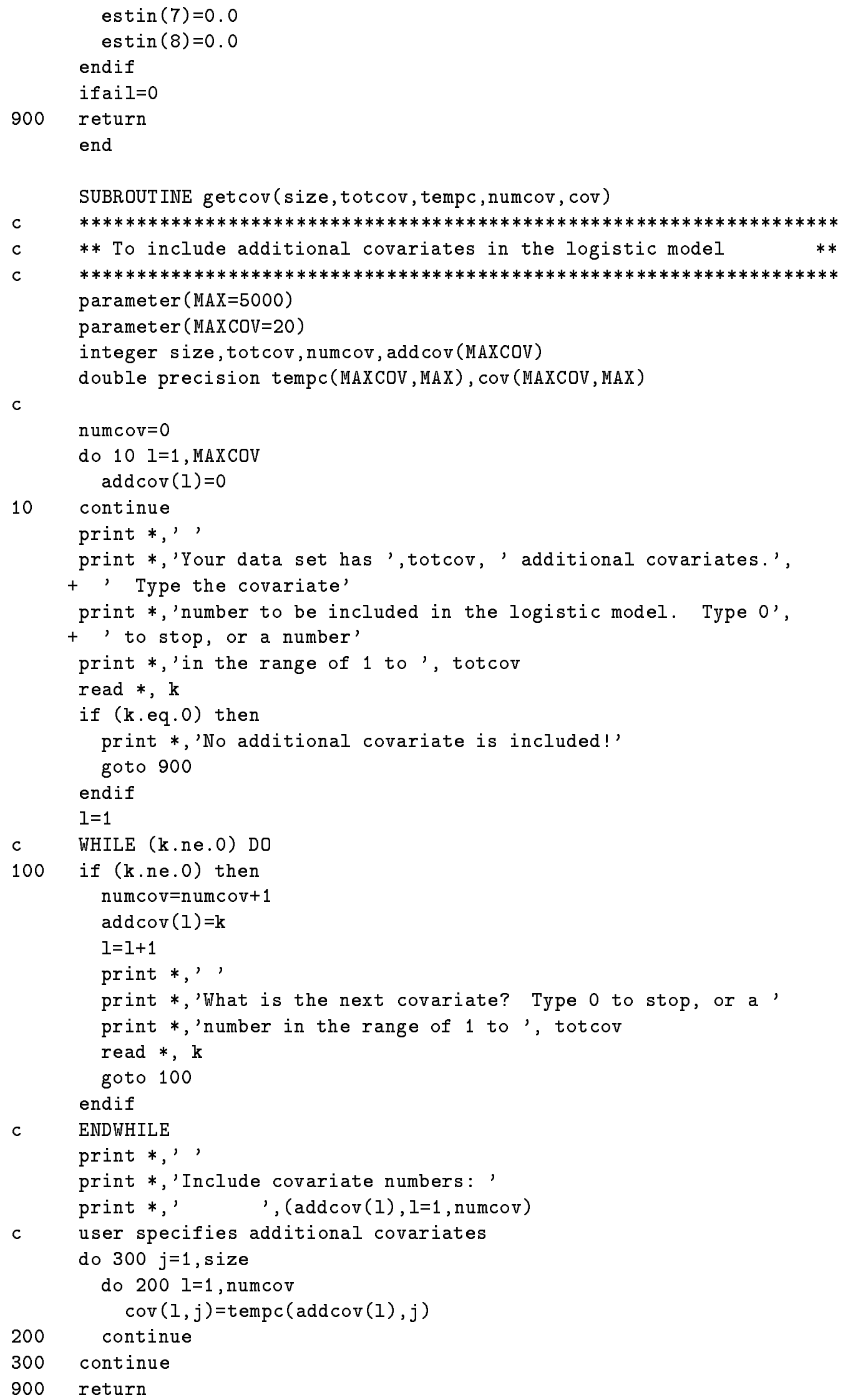


end

SUBROUTINE getest (nboot,model,pi2eq1, size,ftime,age,delta,

+ numcov, cov, estin, estout, llout, coeout, iter, ifail, code)

c

C

C

C

C

300

500

$* * * * * * * * * * * * * * * * * * * * * * * * * * * * * * * * * * * * * * * * * * * * * * * * * * * * * * * * * * * * * * * * * * *$

** To obtain the bootstrap samples and get the final estimates **

integer MB, MAX, MAXCOV

parameter $(\mathrm{MB}=100)$

parameter $(\mathrm{MAX}=5000)$

parameter $($ MAXCOV=20)

integer nboot,model, pi2eq1, size,delta (3,MAX), bdelta (3,MAX)

integer numcov, iter, ifail, code

double precision ftime(MAX), age(MAX), cov(MAXCOV, MAX), estin(8)

double precision bftime(MAX), bage(MAX), bcov(MAXCOV, MAX)

double precision estout (MB,8), llout (MB), coeout (MB, MAXCOV)

if (nboot.eq.1) then

for complete data (nboot.eq.1)

ifail $=2$

CALL mle(nboot, model, pi2eq1, size,ftime, age, delta, numcov, cov,

+ estin, 1, estout, llout, coeout, iter, ifail, code)

else

for (nboot.gt.1)

do $500 \mathrm{k}=1$, nboot

ifail=2

WHILE (ifail.ne.0) DO

if (ifail.ne.0) then

CALL boot (size,ftime, age, delta, numcov, cov, bftime,

$+\quad$ bage, bdelta, bcov)

CALL mle(nboot,model,pi2eq1, size, bftime, bage, bdelta,

$+\quad$ numcov, bcov, estin, $\mathrm{k}$, estout, llout, coeout, iter, ifail, code)

goto 300

endif

ENDWHILE

continue

endif

return

end

SUBROUTINE mle(nboot,model,pi2eq1, size,tj, xj,dj,numcov, $c j$,

+ estin, bnum, estout, llout, coeout, iter, ifail, code)

double precision est (8), newest (8), pi (2, MAX), s (2, MAX), tau(2, MAX) 
double precision coe(MAXCOV), 11 , ddiff

intrinsic abs

C

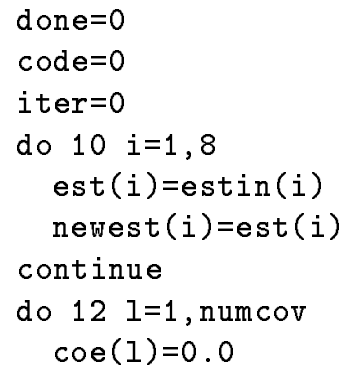




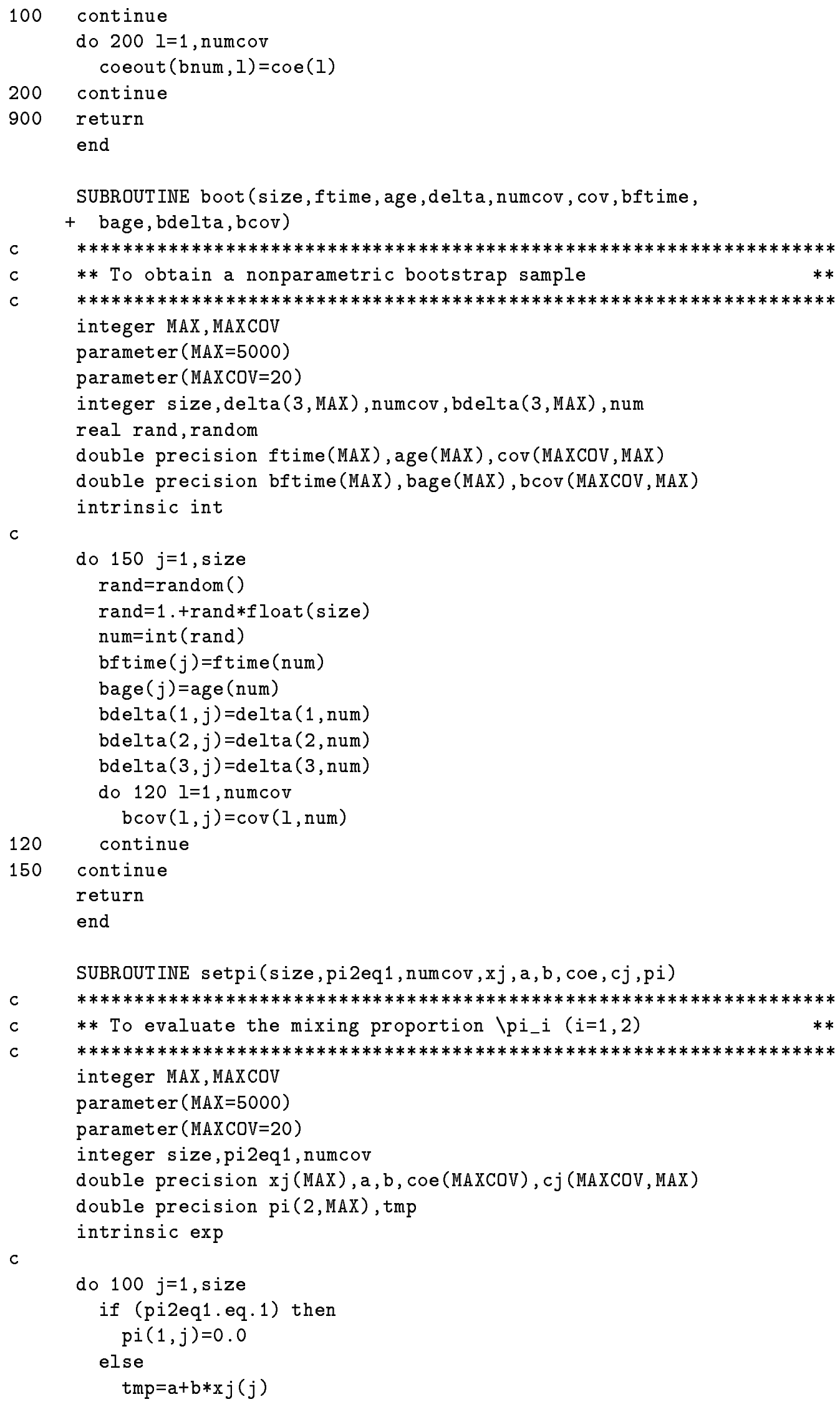


double precision est (8), pi (2, MAX), xj(MAX), cj (MAXCOV, MAX)

double precision coe(MAXCOV), s ( 2, MAX), anew, bnew, tau ( 2, MAX)

double precision $a, b$, newcoe (MAXCOV), ddiff

double precision arr(MAXCOV+2, MAXCOV+2), u(MAXCOV+2)

intrinsic abs

C

ctr $=0$

code $=0$

order $=$ num cov +2

done $=0$

$a=\operatorname{est}(7)$

$\mathrm{b}=\mathrm{est}(8)$

c WHILE (done.ne.1) AND (code.eq.0) DO

10 if (done.ne.1.and.code.eq.0) then

$\operatorname{ddiff}=0$.

CALL caltau (size,pi,s, tau)

CALL setarr (size, a,b, pi, $x j, d j, c j, c o e$, numcov, tau, order, arr, u)

CALL invert (arr, order, code)

if (code.ne.0) goto 100

anew $=\mathrm{a}$

bnew $=\mathrm{b}$

do $20 i=1$, order anew $=\operatorname{anew}+\operatorname{arr}(1, i) * u(i)$

bnew $=$ bnew $+\operatorname{arr}(2, i) * u(i)$

20 continue

do $301=1$, numcov

newcoe $(1)=\operatorname{coe}(1)$

30

continue

do $50 i=1$, order

do $40 \quad 1=1$, numcov

newcoe $(1)=\operatorname{newcoe}(1)+\operatorname{arr}(1+2, i) * u(i)$

40 continue

50 continue

$\operatorname{ddiff}=a b s(a-a n e w)+a b s(b-b n e w)$

do $601=1$, numcov

ddiff=ddifftabs (newcoe(1)-coe(1))

coe $(1)=$ newcoe $(1)$

60

continue

CALL setpi(size,pi2eq1, numcov, $x j$, anew, bnew, newcoe,cj,pi)

$\mathrm{a}=$ anew

$\mathrm{b}=\mathrm{bnew}$

if (ddiff.1t.0.0001) done=1

ctr $=c t r+1$

if (ctr.gt.800 .or. ddiff.gt.15.0) code=2

100

continue

goto 10

endif

c ENDWHILE

if (code.eq.0) then

ifail $=0$

CALL caltau (size,pi,s, tau)

endif

return

end 
SUBROUTINE caltau(size,pi, s, tau)

** To evaluate the posterior probability \tau, using

** pre-calculated values for $\backslash p i_{-} i$ and $s_{-} i(i=1,2)$

integer MAX

parameter $($ MAX $=5000)$

integer size

double precision pi (2, MAX), s (2, MAX), tau (2, MAX), temp

C

do $100 j=1$, size

temp $=p i(1, j) * s(1, j)$

$\operatorname{tau}(1, j)=$ temp $/($ temp $+p i(2, j) * s(2, j))$

$\operatorname{tau}(2, j)=1 \cdot 0-\operatorname{tau}(1, j)$

continue

return

end

SUBROUTINE setarr(size, a, b, pi, xj, dj, cj, coe, numcov, tau, order, arr , u)

integer MAX, MAXCOV

parameter $($ MAX $=5000)$

parameter $(\mathrm{MAXCOV}=20)$

integer size, dj $(3, \mathrm{MAX})$, numcov, order

double precision $a, b, p i(2, \operatorname{MAX}), x j(\operatorname{MAX}), c j$ (MAXCOV, MAX)

double precision coe(MAXCOV), tau (2, MAX)

double precision arr (MAXCOV+2, MAXCOV+2), u(MAXCOV+2)

double precision $\operatorname{varr}(\mathrm{MAX}), \mathrm{xarr}(\mathrm{MAXCOV}+2, \mathrm{MAX}), \mathrm{tmp}, \mathrm{yj}, \mathrm{t}$

c

do $20 i=1$, order

$u(i)=0.0$

do $10 \mathrm{k}=1$, order $\operatorname{arr}(i, k)=0.0$

20 continue

do $40 \mathrm{j}=1$, size

$\operatorname{varr}(j)=\operatorname{pi}(1, j) * \operatorname{pi}(2, j)$

$\operatorname{xarr}(1, j)=1.0$

$\operatorname{xarr}(2, j)=x j(j)$

do $301=1$, numcov

$\operatorname{xarr}(1+2, j)=c j(1, j)$

30 continue

40 continue

do $70 i=1$, order

do $60 \mathrm{k}=1$, order

tmp $=0.0$

do $50 j=1$, size

tmp $=\operatorname{tmp}+\operatorname{xarr}(i, j) * \operatorname{xarr}(k, j) * \operatorname{varr}(j)$

50

continue

$\operatorname{arr}(i, k)=\operatorname{tmp}$

60

continue 
80 continue

C

$\operatorname{ctr}=0$

do $20 i=1$, order

do $10 \mathrm{k}=1, \mathrm{i}$

$\operatorname{ctr}=\operatorname{ctr}+1$

$\operatorname{matrix}(\operatorname{ctr})=\operatorname{arr}(i, k)$

continue

10

continue

nullty $=0$

ifault $=0$

CALL syminv(matrix, order, ctr, inv, work, nullty, ifault)

if (ifault.ne.0) then

code $=1$

goto 900

endif

ctr $=0$

do $40 i=1$, order

do $30 \mathrm{k}=1$, $i$

ctr $=$ ctr +1

$\operatorname{arr}(i, k)=i n v(c t r)$

$\operatorname{arr}(k, i)=i n v(c t r)$

30

continue

40 continue

900 return

end

SUBROUTINE callhy(model, size,tj, xj,dj,est, newest, tau, ifail, code) 
c

code $=0$

indlo $=1$

if (model.eq.2) indlo $=2$ 
return

end

SUBROUTINE evalfn( $\mathrm{n}, \mathrm{x}$, fvec, iflag)

$\mathrm{C}$

** To evaluate the system of functions

$* * * * * * * * * * * * * * * * * * * * * * * * * * * * * * * * * * * * * * * * * * * * * * * * * * * * * * * * * * * * * * * * * * *$

integer MAX

parameter $($ MAX $=5000)$

integer $\mathrm{n}$, iflag

double precision $x(*)$, fvec $(*)$

double precision $t 1, t 2, t 3, d c, t c, h v a l$

double precision tot 1 , tot 2 , beta, gamma, lambda

common/hybr1/hmodel, hsize, hd j, hgroup

integer hmodel,hsize,hdj ( 3 , MAX), hgroup

common/hybr2/htj, hxj,htau

double precision htj(MAX), hxj(MAX), htau(2, MAX)

intrinsic exp

c

bet $a=x(1)$

gamma $=x(2)$

CALL calcl(hmodel,hsize,htj,hxj,hdj,htau, beta, gamma,

+ hgroup, lambda)

tot $1=0.0$

tot $2=0.0$

do $100 \mathrm{j}=1$,hsize

$\mathrm{dc}=\operatorname{hdj}($ hgroup,$j$ )

tc=htau (hgroup, $j$ )

$t 1=\exp ($ lambda+gamma*hxj(j))

$t 2=\exp (\operatorname{beta} * h t j(j))-1.0$

hval $=(\mathrm{t} 1 * \mathrm{t} 2)$

$t 1=(\operatorname{dc}+h d j(3, j) * t c)$

t2 $=-h t j(j) /$ beta*exp $($ lambda+gamma*hxj(j)+beta*htj(j))

t $3=$ hval / (beta*beta)

tot $1=\operatorname{tot} 1+d c * h t j(j)+t 1 *(t 2+t 3)$

$t 1=\mathrm{dc} * \operatorname{hxj}(j)$

$t 2=-(d c+h d j(3, j) * t c)$

$t 3=h x j(j) * h v a l /$ beta

tot $2=$ tot $2+t 1+t 2 * t 3$

100 continue

fvec $(1)=\operatorname{tot} 1$

fvec $(2)=$ tot 2

return

end

SUBROUTINE calcl(model, size,t $j, x j, d j$, tau, beta, gamma, ind, lambda)

c $\quad * * * * * * * * * * * * * * * * * * * * * * * * * * * * * * * * * * * * * * * * * * * * * * * * * * * * * * * * * * * * * * * * * * *$

c $\quad * *$ To solve for lambda given current estimates of beta \& gamma **

c $\quad * * * * * * * * * * * * * * * * * * * * * * * * * * * * * * * * * * * * * * * * * * * * * * * * * * * * * * * * * * * * * * * * * * *$

integer MAX

parameter $(\mathrm{MAX}=5000)$

integer model, size, dj $(3, \mathrm{MAX})$, ind

double precision $t j($ MAX) $, x j(M A X), t a u(2, M A X)$, beta, gamma, lambda

double precision $t, t 1, t 2$ 
C

lambda $=0.0$

if (ind.eq.1.and.model.eq.2) then

bet $a=0.0$

gamma $=0.0$

goto 900

endif

$t 1=0.0$

$\mathrm{t} 2=0.0$

do $10 j=1$, size

t1 $=$ t1 + float $(\operatorname{dj}($ ind,$j))$

$t=\exp (\operatorname{gamma} * x j(j)) *(\exp (\operatorname{beta} * t j(j))-1.0)$

t2 $=$ t $2+($ float $(\operatorname{dj}($ ind,$j))+d j(3, j) * \operatorname{tau}($ ind,$j)) * t$

10

continue

$\operatorname{lambda}=\log ($ beta*t1/t2)

900

return

end

SUBROUTINE findll(model,pi2eq1, size,tj,xj,dj,est,pi,ll)

C

********************************************************************

C $\quad * *$ To evaluate the maximum log likelihood

c $\quad * * * * * * * * * * * * * * * * * * * * * * * * * * * * * * * * * * * * * * * * * * * * * * * * * * * * * * * * * * * * * * * * * * *$

integer MAX

parameter $($ MAX $=5000)$

integer model, pi2eq1, size, dj (3, MAX)

double precision $t j($ MAX), $x j($ MAX), est (8), pi $(2, \operatorname{MAX}), 11$

double precision $t 1, t 2, t 3, t e, t f, m u l, u d r f l$

double precision $11, \mathrm{~g} 1, \mathrm{~b} 1,12, \mathrm{~g} 2, \mathrm{~b} 2, \mathrm{p} 1, \mathrm{p} 2$

intrinsic $\log$

c

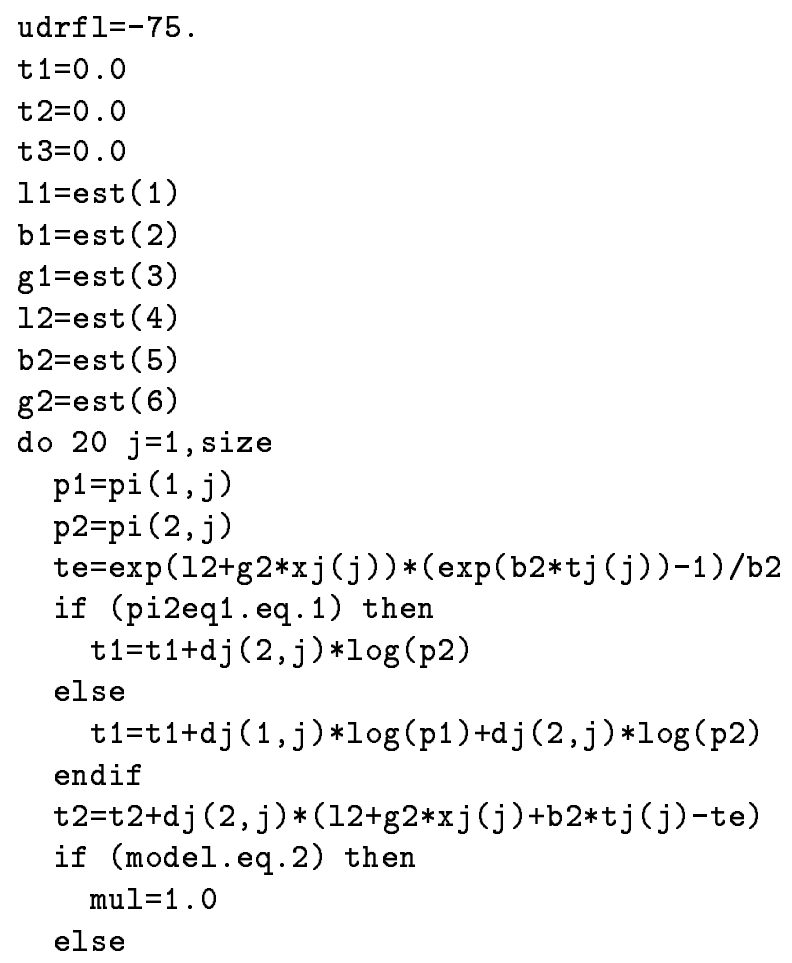




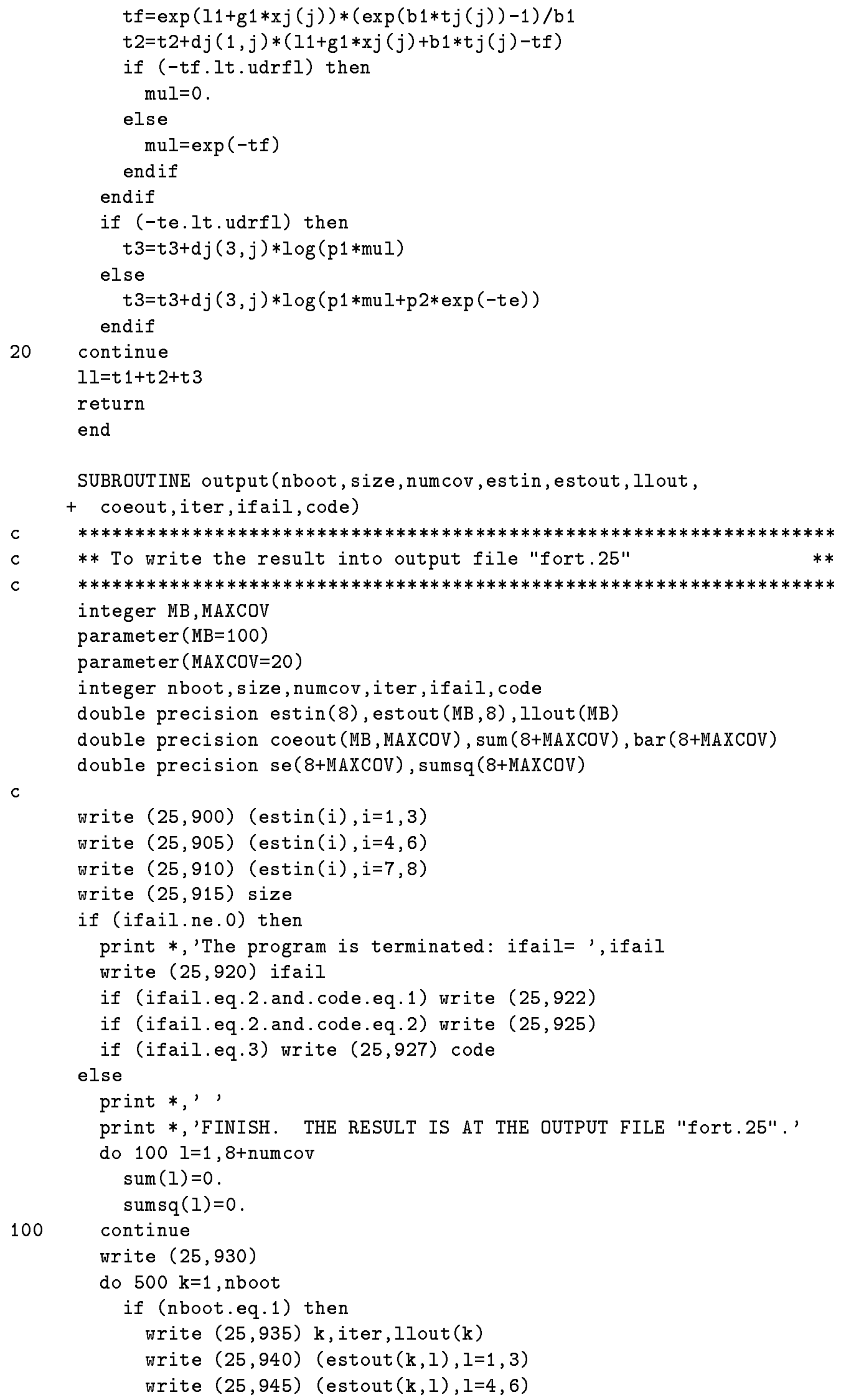




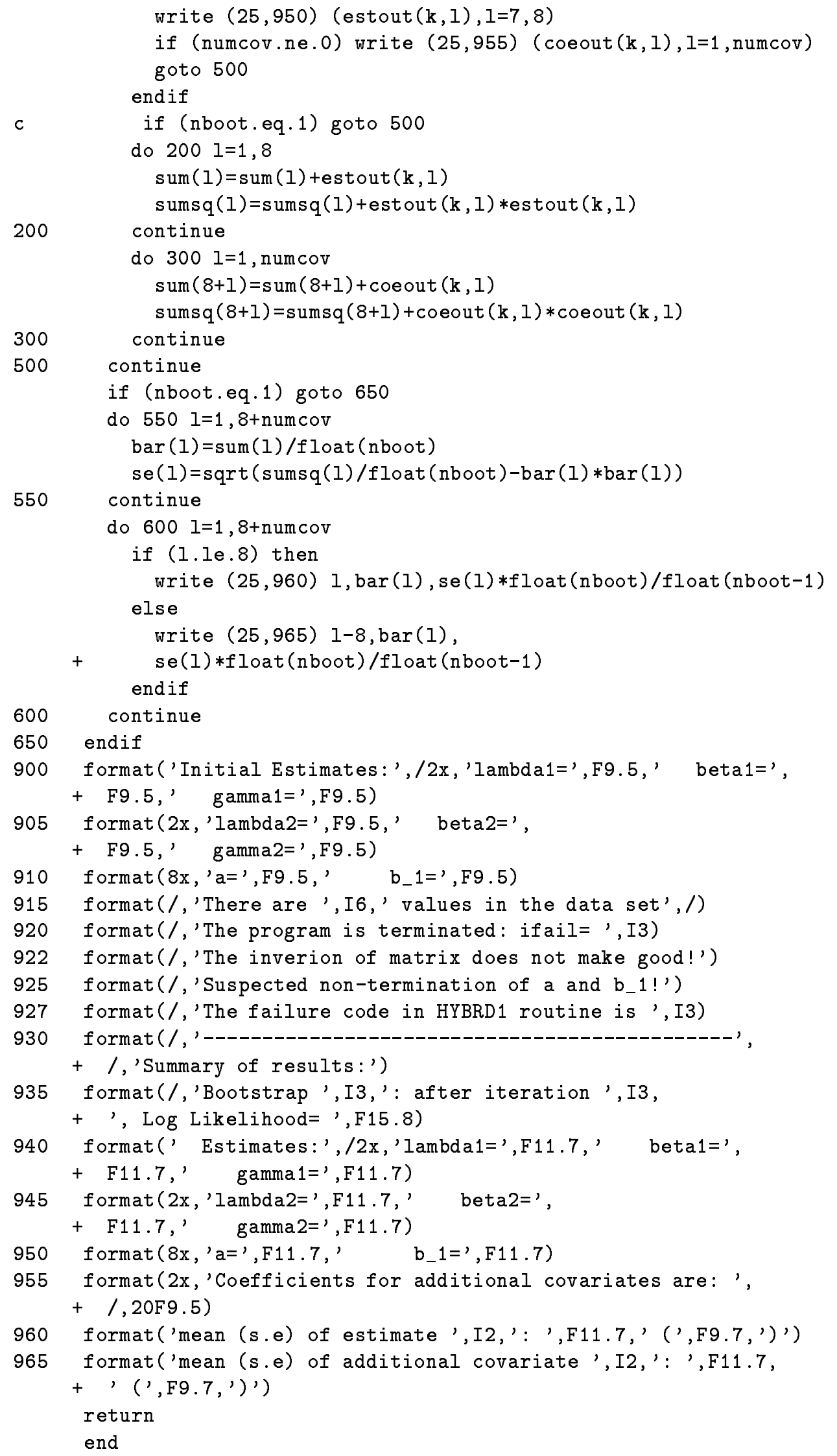




\section{BIBLIOGRAPHY}

Crowley, J. and Hu, M. (1977). Covariance analysis of heart transplant survival data. Journal of the American Statistical Associations 72, 27-36.

Dempster, A.P., Laird, N.M. and Rubin, D.B. (1977). Maximum likelihood from incomplete data via the EM algorithm (with discussion). Journal of the Royal Statistical Society $B$ 39, 1-38.

Efron, B. (1979). Bootstrap methods: another look at the jackknife. Annals of Statistics 7, 1-26.

Efron, B. (1982). The Jackknife, the Bootstrap and Other Resampling Plans. Philadelphia: SIAM.

Farewell, V.T. (1982). The use of mixture models for the analysis of survival data with long-term survivors. Biometrics 38, 1041-1046.

Gordon, N.H. (1990). Maximum likelihood estimation for mixtures of two Gompertz distributions when censoring occurs. Communications in Statistics - Simulation and Computation 19, 733-747.

Gordon, N.H. (1990). Application of the theory of finite mixtures for the estimation of 'cure' rates of treated cancer patients. Statistics in Medicine 9, 397-407.

Kuk, Y.C. (1992). A semiparametric mixture model for the analysis of competing risks data. Australian Journal of Statistics 34, 169-180.

Larson, M.G. and Dinse, G.E. (1985). A mixture model for the regression analysis of competing risks data. Applied Statistics 34, 201-211.

McLachlan, G.J. and Krishnan, T. (1997). The EM algorithm and Extensions. New York: Wiley.

Meng, X.L and Rubin, D.R. (1993). Maximum likelihood estimation via the ECM algorithm: a general framework. Biometrika 80, 267-278.

Moré, J.J., Garbow, B.S. and Hillstrom, K.E. (1980). User Guide for MINPACK-1, ANL-80-74. Argonne National Laboratory, Chicago. 